\title{
PERIODIC ORBITS OF THE SPATIAL ANISOTROPIC MANEV PROBLEM
}

\author{
JAUME LLIBRE $^{1}$ AND AMMAR MAKHLOUF ${ }^{2}$
}

\begin{abstract}
We study the periodic orbits of the spatial anisotropic Manev problem which depend on three parameters.
\end{abstract}

\section{Introduction AND STATEMENT OF THE MAIN RESUlts}

The objective of this paper is to study the periodic orbits of the spatial anisotropic Manev problem given by the Hamiltonian

$$
\mathrm{H}=\frac{1}{2}\left(p_{x}^{2}+p_{y}^{2}+p_{z}^{2}\right)-\frac{1}{\sqrt{\mu\left(x^{2}+y^{2}\right)+z^{2}}}-\frac{\varepsilon \beta}{\mu\left(x^{2}+y^{2}\right)+z^{2}},
$$

where $\mu$ is near $1, \beta \neq 0$ is a parameter and $\varepsilon$ is small.

The dynamics of the planar anisotropic Manev problem was studied in [4] and [6]-[8], including information on its periodic orbits, but as far as we know there are no works on the spatial periodic orbits of the Manev problem.

If $\mu=1$ and $\beta=0$, we have the spatial Kepler problem, see for instance the book [3].

If $\mu \neq 1$ and $\beta=0$, we have the spatial anisotropic Manev problem, which originally comes from the quantum mechanics, see for instance $[10,1,2]$.

If $\mu=1$ and $\beta \neq 0$ then we have the spatial Manev problem. One of the advantages of the Manev problem over the Keplerian is that it explains the perihelion advance of the inner planets with the same accuracy as relativity, see [5], [9], [11], [12]-[15] and [17]-[19].

Note that the Hamiltonian (1) is symmetric with respect to the z-axis. Then it is easy to check that the third component $K=x p_{y}-y p_{x}$ of the angular momentum is a first integral of the Hamiltonian system with Hamiltonian (1). We shall use this integral $K$ to simplify the analysis of the given axially symmetric perturbed system.

\footnotetext{
Key words and phrases. Periodic orbits, perturbed Kepler problem, spatial anisotropic Manev problem.

2010 Mathematics Subject Classification. Primary: 70F05, 37C27, 37J30. Secondary: 70F15, 37J25.
} 
Since $\mu$ is near 1 and $\varepsilon$ is small we take $\mu=1-\varepsilon$, and doing Taylor series in $\varepsilon$ at $\varepsilon=0$ of the Hamiltonian (1), we obtain

(2)

$\mathrm{H}=\frac{1}{2}\left(p_{x}^{2}+p_{y}^{2}+p_{z}^{2}\right)-\frac{1}{\sqrt{x^{2}+y^{2}+z^{2}}}-\varepsilon \frac{x^{2}+y^{2}+2 \sqrt{x^{2}+y^{2}+z^{2}} \beta}{2\left(x^{2}+y^{2}+z^{2}\right)^{3 / 2}}+O\left(\varepsilon^{2}\right)$.

In the following we shall use the Delaunay variables for studying easily the periodic orbits of the Hamiltonian system associated to the Hamiltonian (2), see $[3,16]$ or section 2 for more details on the Delaunay variables. Thus in Delaunay variables the Hamiltonian (2) has the form

$$
\mathrm{H}=-\frac{1}{2 L^{2}}+\varepsilon P(l, g, k, L, G, K)+O\left(\varepsilon^{2}\right),
$$

where $P(l, g, k, L, G, K)$ is equal to

$$
\frac{-(G+L)\left(\left(G^{2}+K^{2}\right) L+4 G \beta\right)+\left(G^{2}-K^{2}\right)(G-L) L \cos (2 g)}{4 G^{2} L^{3}(G+L)},
$$

where $l$ is the mean anomaly, $g$ is the argument of the perigee of the unperturbed elliptic orbit measured in the invariant plane, $k$ is the longitude of the node, $L$ is the square root of the semi-major axis of the unperturbed elliptic orbit, $G$ is the modulus of the total angular momentum, and $K$ is the third component of the angular momentum.

Our main result is the following one.

Theorem 1. On every energy level $H=h<0$ and for the third component of the angular momentum $K=0$, the spatial anisotropic Kepler problem given by the Hamiltonian (2) for $\varepsilon \neq 0$ sufficiently small and for every $k_{0} \in[0,2 \pi)$ has:

(a) Two $2 \pi$-periodic solutions $\gamma_{\varepsilon}(l)=(g(l, \varepsilon), k(l, \varepsilon), L(l, \varepsilon), G(l, \varepsilon), K(l, \varepsilon))$ such that

$\gamma_{\varepsilon}(0) \rightarrow\left( \pm \frac{1}{2} \arccos (16 h \beta), k_{0}, \frac{1}{\sqrt{-2 h}}, \frac{1}{\sqrt{-2 h}}, 0\right)$ when $\varepsilon \rightarrow 0$,

if $|\beta|<1 /|16 h|$.

(b) Two $2 \pi$-periodic solution $\gamma_{\varepsilon}(l)$ such that

$$
\begin{aligned}
& \gamma_{\varepsilon}(0) \rightarrow\left( \pm \frac{\pi}{2}, k_{0}, \frac{1}{\sqrt{-2 h}}, \frac{2 \sqrt{-2 h} \beta+\sqrt{2 \beta}}{1+4 h \beta}, 0\right) \text { when } \varepsilon \rightarrow 0, \\
& \text { if } \beta>0 \text { and }(1+4 h \beta)(1-2 \sqrt{-h \beta}+8 h \beta) \neq 0 .
\end{aligned}
$$

(c) Two $2 \pi$-periodic solution $\gamma_{\varepsilon}(l)$ such that

$$
\begin{aligned}
& \gamma_{\varepsilon}(0) \rightarrow\left( \pm \frac{\pi}{2}, k_{0}, \frac{1}{\sqrt{-2 h}}, \frac{2 \sqrt{-2 h} \beta-\sqrt{2 \beta}}{1+4 h \beta}, 0\right) \text { when } \varepsilon \rightarrow 0, \\
& \text { if } \beta>0 \text { and }(1+4 h \beta)(1+2 \sqrt{-h \beta}+8 h \beta) \neq 0 .
\end{aligned}
$$


(d) Two $2 \pi$-periodic solution $\gamma_{\varepsilon}(l)$ such that

$$
\begin{aligned}
& \gamma_{\varepsilon}(0) \rightarrow\left(\left\{\begin{array}{l}
0 \\
\pi
\end{array}\right\}, k_{0}, \frac{1}{\sqrt{-2 h}}, \frac{2 \sqrt{-2 h} \beta+\sqrt{-2 \beta}}{-1+4 h \beta}, 0\right) \text { when } \varepsilon \rightarrow 0, \\
& \text { if } \beta<0 \text { and }(1-4 h \beta)(1-4 \sqrt{h \beta}+4 h \beta)(-1-2 \sqrt{h \beta}+8 h \beta) \neq 0 .
\end{aligned}
$$

(e) Two $2 \pi-$ periodic solution $\gamma_{\varepsilon}(l)$ such that

$$
\begin{aligned}
& \gamma_{\varepsilon}(0) \rightarrow\left(\left\{\begin{array}{l}
0 \\
\pi
\end{array}\right\}, k_{0}, \frac{1}{\sqrt{-2 h}}, \frac{2 \sqrt{-2 h} \beta-\sqrt{-2 \beta}}{-1+4 h \beta}, 0\right) \text { when } \varepsilon \rightarrow 0, \\
& \text { if } \beta<0 \text { and }(1-4 h \beta)(-1+2 \sqrt{h \beta}+8 h \beta)(1+4 \sqrt{h \beta}+4 h \beta) \neq 0 .
\end{aligned}
$$

Theorem 1 is proved in section 3 .

We note, as we shall see in the proof of Theorem 1, that the averaging method that we shall apply for finding the periodic solutions of Theorem 1 only find periodic solutions when the first integral $K=0$. See the appendix for a summary of the averaging method used here.

\section{Delauney variables}

The transformation of Delauney is given by

$$
\begin{aligned}
& x=r(\cos (f+g) \cos k-c \sin (f+g) \sin k), \\
& y=r(\cos (f+g) \sin k+c \sin (f+g) \cos k), \\
& z=r s \sin (f+g),
\end{aligned}
$$

with $c=K / G$ and $s^{2}=1-K^{2} / G^{2}$. The true anomaly $f$ and the eccentric anomaly $E$ are auxiliary quantities defined by the relations

$$
\begin{gathered}
\sqrt{1-e^{2}}=\frac{G}{L}, \quad r=(1-e \cos E), \quad l=E-e \sin E . \\
\sin f=\frac{a \sqrt{1-e^{2}} \sin E}{r}, \quad \cos f=\frac{a(\cos E-e)}{r},
\end{gathered}
$$

where $e$ is the eccentricity of the unperturbed elliptic orbit. Note that the angular variable $k$ is a cyclic variable for the Hamiltonian (3), and consequently $K$ is a first integral of the Hamiltonian system as we already knew.

\section{Proof of Theorem 1}

We shall write the Hamiltonian system on the energy level $H=h<0$. From the equation $H=h$ we isolate $L=1 / \sqrt{-2 h}+O(\varepsilon)$. So the Hamiltonian 
system with Hamiltonian (3) eliminating $L$ becomes

$$
\begin{aligned}
\frac{d G}{d t}= & \varepsilon \frac{(1-\sqrt{-2 h} G) h\left(G^{2}-K^{2}\right) \sin (2 g)}{G^{2}(1+\sqrt{-2 h} G)}+O\left(\varepsilon^{2}\right), \\
\frac{d K}{d t}= & 0, \\
\frac{d l}{d t}= & 2 \sqrt{2}(-h)^{3 / 2}+O(\varepsilon), \\
\frac{d g}{d t}= & \frac{2 h}{G^{3}(2 \sqrt{-h} G+\sqrt{2})^{2}}\left(\left(2 h G^{2}-2 \sqrt{-2 h} G-1\right) K^{2}+\right. \\
& 2 G(2 G(2+\sqrt{-2 h} G) h-\sqrt{-2 h}) \beta+ \\
\frac{d k}{d t}= & \varepsilon \frac{\left.h K\left(2 h K^{2} G^{2}-\sqrt{-2 h} G^{3}+\sqrt{-2 h} K^{2} G+K^{2}\right) \cos (2 g)\right)+O\left(\varepsilon^{2}\right),}{G^{2}(2 \sqrt{-h} G+\sqrt{2})}
\end{aligned}
$$

Now we take $l$ as new independent variable, and the previous equations of motion restricted to $K=\bar{K}$ write

$$
\begin{aligned}
\frac{d G}{d l}=-\varepsilon \frac{(1-\sqrt{-2 h} G)\left(G^{2}-\bar{K}^{2}\right) \sin (2 g)}{2 \sqrt{-2 h} G^{2}(1+\sqrt{-2 h} G)}+O\left(\varepsilon^{2}\right), \\
\frac{d g}{d t}=-\varepsilon \frac{1}{\sqrt{-2 h} G^{3}(2 \sqrt{-h} G+\sqrt{2})^{2}}\left(\left(2 h G^{2}-2 \sqrt{-2 h} G-1\right) \bar{K}^{2}+\right. \\
\left.\left(2 h \bar{K}^{2} G^{2}-\sqrt{-2 h} G^{3}+\sqrt{-2 h \bar{K}^{2}} G+\bar{K}^{2}\right) \cos (2 g)\right)+ \\
2 G(2 G(2+\sqrt{-2 h} G) h-\sqrt{-2 h}) \beta+O\left(\varepsilon^{2}\right), \\
\frac{d k}{d t}=-\varepsilon \frac{\bar{K}(2 \sqrt{-h} G-(\sqrt{2}-2 G \sqrt{-h}) \cos (2 g)+\sqrt{2})}{2 \sqrt{-2 h} G^{2}(2 \sqrt{-h} G+\sqrt{2})}+O\left(\varepsilon^{2}\right) .
\end{aligned}
$$

Since in the previous differential system the right hand side functions do not depend on the angular variable $l$, its averaged equations with respect to $l$ are the same but without $O\left(\varepsilon^{2}\right)$. Therefore, from the appendix we have that the equilibrium points of the averaged equations which have non-zero Jacobian will provide periodic solutions of the Hamiltonian system with Hamiltonian (3) in the energy level $H=h<0$ and in the level $K=\bar{K}$. Now we shall compute these equilibria.

From the first equation of system (5) we have that $(1-\sqrt{-2 h} G)\left(G^{2}-\bar{K}^{2}\right)$ $\sin (2 g)=0$. So we separate the computation of the equilibria in three cases. 
Case 1: $1-\sqrt{-2 h} G=0$. This implies that $G=1 / \sqrt{-2 h}$. Then the equilibrium points must satisfy the remaining two equations of system (5), i.e.

$$
\frac{1}{8}\left(-8 h\left(\bar{K}^{2}+2 \beta\right)+\left(1+2 h \bar{K}^{2}\right) \cos (2 g)\right)=0, \quad-\frac{1}{\sqrt{2}}(-h)^{1 / 2} \bar{K}=0 .
$$

Therefore $\bar{K}=0$, we obtain the equation $-2 h \beta+\cos (2 g) / 8=0$. It possesses the solutions $g= \pm \frac{1}{2} \arccos (16 h \beta)$, which exist when $16 h \beta \in[-1,1]$, or equivalently $|\beta| \leq 1 /|16 h|$. Therefore we have the two solutions $(g, G, \bar{K})=$ $( \pm \arccos (16 h \beta) / 2,1 / \sqrt{-2 h}, 0)$. The Jacobian of the averaged equations at these two solutions is

$$
\frac{\sqrt{-h}\left(1-256 h^{2} \beta^{2}\right)}{16 \sqrt{2}}
$$

Then, from the appendix, statement (a) of Theorem 1 is proved.

Case 2: $G= \pm \bar{K}$. First we consider $G=\bar{K}$. The last two equations from the averaged system becomes

$$
\begin{aligned}
& \frac{\left(1+2 h \bar{K}^{2}\right)(4 h \beta-\sqrt{-2 h K})+\bar{K}\left((-2 h)^{3 / 2} \bar{K}^{2}+4 h \bar{K}+\sqrt{-2 h}\right) \cos (2 g)}{4 h \bar{K}^{2}\left(2 h \bar{K}^{2}+1\right)}=0, \\
& \frac{2 \sqrt{-h} \bar{K}-(\sqrt{2}-2 \sqrt{-h} \bar{K}) \cos (2 g)+\sqrt{2}}{4 \bar{K}(\sqrt{2} h \bar{K}-\sqrt{-h})}=0 .
\end{aligned}
$$

From the last equation we deduce $\bar{K}=\frac{\cos (2 g)-1}{2 \sqrt{-2 h} \cos ^{2} g}$. We then obtain $-2 h \beta \cot ^{4} g=0$. This equation has the two solutions $g= \pm \pi / 2$. But then $\bar{K}$ is not defined at these two values of $g$. So, no equilibria when $G=\bar{K}$.

In a similar way we can see that the case $G=-\bar{K}$ does not provide equilibria of the averaged system.

Case 3: $\sin (2 g)=0$. This equation has the three solutions $g=0, \pm \frac{\pi}{2}, \pi$. We consider three subcases.

Subcase A: $g=\pi / 2$. Then the last two equations from the averaged system becomes

$$
\begin{aligned}
& \frac{G h\left(3 \bar{K}^{2}+2 \beta-G(4 h \beta G+G-4 \sqrt{-2 h} \beta)\right)-\sqrt{-2 h} \bar{K}^{2}}{G^{3}(2 \sqrt{-h} G+\sqrt{2})^{2} h}=0, \\
& \frac{\bar{K}}{G^{2}(2 G h-\sqrt{2} \sqrt{-h})}=0 .
\end{aligned}
$$


From the last equation we deduce $\bar{K}=0$. Then the second equation from the averaged system reduces to

$$
\frac{2 \beta-G(4 h \beta G+G-4 \sqrt{-2 h} \beta)}{G^{2}(2 \sqrt{-h} G+\sqrt{2})^{2}}=0 .
$$

Solving this last equation with respect to $G$ we get

$$
G=\frac{2 \sqrt{-2 h} \beta \pm \sqrt{2 \beta}}{1+4 h \beta} .
$$

Therefore we have the two solutions

$$
\left(g, G_{ \pm}, \bar{K}\right)=\left(\frac{\pi}{2}, \frac{2 \sqrt{-2 h} \beta \pm \sqrt{2 \beta}}{1+4 h \beta}, 0\right) .
$$

The Jacobian of the averaged equations at the solution $\left(g, G_{+}, \bar{K}\right)$ is

$$
\frac{(1+4 h \beta)^{7}(1-2 \sqrt{-h \beta}+8 h \beta)}{4 \sqrt{2} h(1+2 \sqrt{-h \beta})^{8} \beta^{3 / 2}},
$$

and at the solution $\left(g, G_{-}, \bar{K}\right)$ is

$$
-\frac{(1+4 h \beta)^{7}(1+2 \sqrt{-h \beta}+8 h \beta)}{4 \sqrt{2} h(1-2 \sqrt{-h \beta})^{8} \beta^{3 / 2}} .
$$

Then, from the appendix, statements (b) and (c) of Theorem 1 are proved when $g=\pi / 2$.

Subcase B: $g=-\pi / 2$. The proof of statements (b) and (c) of Theorem 1 when $g=-\pi / 2$ are completely similar to case $g=\pi / 2$.

Subcase C: $g=0$, Now the last two equations from the averaged system becomes

$$
\begin{aligned}
& \frac{\bar{K}^{2}+2 \beta+G\left(-4 h \beta G+G+2 \sqrt{-2 h}\left(\bar{K}^{2}+2 \beta\right)\right)}{G^{2}(2 \sqrt{-h} G+\sqrt{2})^{2}}=0, \\
& -\frac{\bar{K}}{\sqrt{-2 h} G^{2}+G}=0 .
\end{aligned}
$$

From this last equation we deduce $\bar{K}=0$. Hence the second equation from the averaged system becomes

$$
\frac{2 \beta+G(-4 h \beta G+G+4 \sqrt{-2 h} \beta)}{G^{2}(2 \sqrt{-h} G+\sqrt{2})^{2}}=0 .
$$

Solving this last equation with respect to $G$ we get

$$
G=\frac{2 \sqrt{-2 h} \beta \pm \sqrt{2 \beta}}{-1+4 h \beta} .
$$


Therefore we have the two solutions

$$
\left(g, G_{ \pm}, \bar{K}\right)=\left(\frac{\pi}{2}, \frac{2 \sqrt{-2 h} \beta \pm \sqrt{2 \beta}}{-1+4 h \beta}, 0\right) .
$$

The Jacobian of the averaged equations at the solution $\left(g, G_{+}, \bar{K}\right)$ is

$$
\frac{\sqrt{-h} \beta(1+2 \sqrt{h \beta}-8 h \beta)(1-4 h \beta)^{6}(-1+4 \sqrt{h \beta}-4 h \beta)}{2 \sqrt{2}(2 \sqrt{h \beta}-1)^{3}(2 h \sqrt{-\beta}+\sqrt{-h})^{2}(2 \sqrt{-h} \beta+\sqrt{-\beta})^{4}},
$$

and at the solution $\left(g, G_{-}, \bar{K}\right)$ is

$$
-\frac{\sqrt{-h} \beta(1-4 h \beta)^{6}(1+4 \sqrt{h \beta}+4 h \beta)(-1+2 \sqrt{h \beta+8 h \beta})}{2 \sqrt{2}(1+2 \sqrt{h \beta})^{3}(\sqrt{-h}-2 h \sqrt{-\beta})^{2}(\sqrt{-\beta}-2 \sqrt{-h} \beta)^{4}} .
$$

Then, from the appendix, statements (d) and (e) of Theorem 1 are proved when $g=0$.

Subcase D: $g=\pi$. The proof of statements (d) and (e) of Theorem 1 when $g=\pi$ are completely similar to case $g=0$.

In short, Theorem 1 is proved.

\section{APPENDIX}

Now we shall present the basic results from averaging theory that we need for proving the results of this paper.

The next theorem provides a first order approximation for the periodic solutions of a periodic differential system, for the proof see Theorems 11.5 and 11.6 of Verhulst [20].

Consider the differential equation

$$
\dot{x}=\varepsilon F(t, x)+\varepsilon^{2} R(t, x, \varepsilon), x(0)=x_{0},
$$

with $x \in D$ where $D$ is an open subset of $\mathbb{R}^{n}$, and $t \geq 0$. Moreover we assume that $F(t, \mathrm{x})$ is $T$ periodic in $t$. Separately we consider in $D$ the averaged differential equation

$$
\dot{y}=\varepsilon f(y), y(0)=x_{0},
$$

where

$$
f(y)=\frac{1}{T} \int_{0}^{T} F(t, y) d t
$$

Under certain conditions, see the next theorem, equilibrium solutions of the averaged equation turn out to correspond with $T$-periodic solutions of equation (7).

Theorem 2. Consider the two initial value problems (6) and (7). Suppose:

(i) F, its Jacobian $\partial F / \partial x$, its Hessian $\partial^{2} F / \partial x^{2}$ are defined, continuous and bounded by an independent constant $\varepsilon$ in $[0, \infty) \times D$ and $\varepsilon \in\left(0, \varepsilon_{0}\right]$.

(ii) $F$ is $T$-periodic in $t$ ( $T$ independent of $\varepsilon$ ). 
(iii) $y(t)$ belongs to $D$ on the interval of time $[0,1 / \varepsilon]$.

Then the following statements hold.

(a) For $t \in[0,1 / \varepsilon]$ we have that $x(t)-y(t)=O(\varepsilon)$, as $\varepsilon \rightarrow 0$.

(b) If $p$ is a equilibrium point of the averaged equation (7) and

$$
\left.\operatorname{det}\left(\frac{\partial f}{\partial y}\right)\right|_{\mathrm{y}=p} \neq 0
$$

then there exists a $T$-periodic solution $\varphi(t, \varepsilon)$ of equation (6) such that $\varphi(0, \varepsilon) \rightarrow p$ as $\varepsilon \rightarrow 0$.

(c) The stability or instability of the periodic solution $\varphi(t, \varepsilon)$ is given by the stability or instability of the equilibrium point $p$ of the averaged system (7). In fact, the equilibrium point $p$ has the stability behavior of the Poincaré map associated to the periodic solution $\varphi(t, \varepsilon)$.

\section{ACKNOWLEDGEMENTS}

The first author was partially supported by MICINN/FEDER grant number MTM2008-03437, AGAUR grant number 2009 SGR 410, ICREA Academia and FP7-PEOPLE-2012-IRSES-316338.

\section{REFERENCES}

[1] J. CASASAyas, And J. Llibre, Qualitative analysis of the anisotropic Kepler problem, Mem. Amer. Math. Soc. 52, no. 312, (1984).

[2] G. Contopoulos And M. Harsoula, Stability and instability in the anisotropic Kepler problem, J. Physics A 38 (2005), 8897-8920.

[3] B. Cordani, The Kepler problem, Progress in Mathematical Physics 29, SpringerVerlag, 2003.

[4] S. Craig, F.N. Diacu, E.A. Lacomba, E. Perez, On the anisotropic Manev problem, J. Math. Phys. 40 (1999), 1359-1375.

[5] J. Delgado, F. Diacu, E.A. Lacomba, A. Mingarelli, Angelo, V. Mioc, E. Perez and C. Stoica, The global flow of the Manev problem, J. Math. Phys. 37 (1996), $2748-2761$.

[6] F. Diacu, Stability in the anisotropic Manev problem, J. Phys. A 33 (2000), 6573-6578.

[7] F. Diacu And M. Santoprete, Nonintegrability and chaos in the anisotropic Manev problem, Phys. D 156 (2001), 39-52.

[8] F. Diacu And M. Santoprete, On the global dynamics of the anisotropic Manev problem, Phys. D 194 (2004), 75-94.

[9] A. Einstein, On the Quantum Theorem of Sommerfeld and Epstein, Deutsche Physikalische Gesellschaft, Verhandlungen 19 (1917), 82-92.

[10] M. Gutzwiller And C. Martin, The quantization of a classically ergodic system. Classical quantum models and arithmetic problems, 287-351, Lecture Notes in Pure and Appl. Math. 92, Dekker, New York, 1984.

[11] J. Llibre, A.E. Teruel, C. Valls And A. De la Fuente, Phase portraits of the Manev systems, J. Physics A 34 (2001), 1919-1934.

[12] G. Maneff, La gravitation et le principe de l'égalité de l'action et de la réaction, Comptes rendus 178 (1924), 2159-2161.

[13] G. Maneff, Die Gravitation und das Prinzip von Wirkung und Gegenwirkung, Zeitschrift für Physik 31 (1925), 786-802. 
[14] G. Maneff, Le principe de la moindre action et la gravitation, C. R. Acad. Sci. Paris Sér. I Math. 190 (1930), 963-965.

[15] G. Maneff, La gravitation et l'énergie au zéro, Comptes rendus 190 (1930), 1374-1377.

[16] K.R. Meyer, G.R. Hall and D. Offin, Introduction to Hamiltonian dynamical systems and the $N$-body problem, Applied Mathematical Sciences 90, Springer New York, 2009.

[17] V. Mioc And C. Stoica, Discussion et résolution complète du problème des deux corps dans le champ gravitationnel de Maneff, C. R. Acad. Sci. Paris Sér. I Math. 320 (1995), 645-648.

[18] V. Mioc and C. Stoica, Discussion et résolution complète du problème des deux corps dans le champ gravitationnel de Maneff (II), C. R. Acad. Sci. Paris Sér. I Math. 321 (1995), 961-964.

[19] F. Szenkovits, V. Mioc and C. Stoica, The Manev-type problems: a topological view, Mathematica 41(64) (1999), 105-120.

[20] F. Verhulst, Nonlinear Differential Equations and Dynamical Systems, Universitext, Springer, 1991.

1 Departament de Matemàtiques, Universitat Autònoma de Barcelona, Bellaterra, 08193-Barcelona, Catalonia, Spain

E-mail address: jllibre@mat.uab.cat

2 Department of mathematics, University Badji Mokhtar, Annaba, Algeria

E-mail address: makhloufamar@yahoo.fr 